\title{
Comparação dos Conteúdos do POSCOMP com o Currículo de Referência dos Cursos de Computação da SBC
}

\author{
Acsa L. A. Augusto, Breno M. F. Viana, Débora E. C. Oliveira \\ Gustavo A. Bezerra, João M. L. Neto, Kevin W. de S. Maciel \\ Patrícia C. Ferraz, Raul S. Silva, Umberto S. Costa \\ Departamento de Matemática e Informática Aplicada - \\ Universidade Federal do Rio Grande do Norte (UFRN) \\ Campus Universitário - Lagoa Nova, \\ Caixa Postal 1524 - 59078-970 - Natal - RN - Brasil \\ \{acsalaiane,bmfviana, debaemili, gustavoalvesbezerra\}@gmail.com \\ \{mendeslopes.joao, kw199918, pcruz.pat, raul95\}@gmail.com \\ \{umberto.costa\}@ufrn.br
}

\begin{abstract}
The National Exam for Post Graduation in Computing (POSCOMP) is an evaluation organized by the Brazilian Computer Society (SBC) whose objective is to grade the Computer Science alumni. This paper proposes a comparative analysis of the POSCOMP editions, from 2014 to 2019, with the SBC's National Curriculum (NC) homologated in 2016. Based on this comparison, we noticed: (i) approximately $60 \%$ of the NC content is not covered in the exams; (ii) only 14 contents have significant and continuous incidences in the exams; (iii) the competence axis defined by the NC have significantly different numbers of corresponding questions explored along the editions of the exam.
\end{abstract}

Resumo. O Exame Nacional para Ingresso na Pós-Graduação em Computação (POSCOMP) é uma avaliação organizada pela Sociedade Brasileira de Computação (SBC) cujo objetivo é avaliar o egresso de Computação. Este trabalho apresenta uma análise comparativa das edições de 2014 a 2019 do POSCOMP com o Currículo de Referência (CR) da SBC homologado em 2016. A partir dessa comparação, foram observados: (i) a ausência de aproximadamente $60 \%$ dos conteúdos do CR nos exames; (ii) apenas 14 conteúdos apresentam incidências significativas e contínuas nos exames; (iii) os eixos de formação definidos pelo CR têm diferenças significativas nos números de questões correspondentes que foram exploradas ao longo das edições do exame.

\section{Introdução}

O Exame Nacional para Ingresso na Pós-Graduação em Computação (POSCOMP) é uma avaliação organizada pela Sociedade Brasileira de Computação (SBC) com o objetivo de avaliar o conhecimento do egresso de Computação. A nota do exame é considerada em processos de seleção para cursos de Pós-Graduação em Computação de diversas universidades no Brasil, como uma etapa eliminatória ou como critério de desempate [SBC 2018]. Dada a relevância do POSCOMP, em [Junior and Brancher 2014] os autores apresentam os resultados de uma pesquisa de opinião de diversos profissionais da Computação sobre 
a relevância dos conteúdos deste exame. Por outro lado, o presente documento apresenta outra análise do POSCOMP, comparando seu conteúdo com aqueles indicados pelo Currículo de Referência (CR) do curso de Ciência da Computação sugerido pela SBC.

A análise apresentada neste artigo surgiu como uma atividade do grupo PET de Ciência da Computação da UFRN a fim de fornecer diretrizes que auxiliassem os alunos que vão prestar o POSCOMP. De modo a identificar o perfil da prova, é feita a investigação de quais conteúdos do CR são mais frequentes e quais não foram explorados, além do que o aplicante deve esperar, indicando o grau de adesão do exame ao CR. A análise contempla as edições de 2014 a 2019 (excluindo 2020 e 2021 pois, devido à pandemia da COVID-19, não houve provas nesses anos). As conclusões dessa análise poderão auxiliar alunos, elaboradores de questões, professores e coordenadores de cursos de graduação: os alunos de graduação poderão direcionar seus esforços ao estudo dos assuntos mais frequentes; os elaboradores de questões poderão, caso considerarem necessário, considerar conteúdos pouco ou não abordados nos exames de até então; os professores poderão revisar os assuntos das disciplinas; por fim, os coordenadores, poderão reestruturar a grade curricular do curso, se necessário, de forma a melhor preparar seus alunos para o exame.

A estrutura deste documento é descrita a seguir. Na Seção 2, o Currículo de Referência desenvolvido pela SBC para o curso de Ciência da Computação no Brasil é apresentado. A Seção 3 apresenta uma visão geral do POSCOMP, abordando sua origem, objetivos e estrutura da avaliação. Na Seção 4, a metodologia de análise comparativa entre a prova e o CR é apresentada. Na Seção 5, são apresentados e discutidos os resultados da análise. Por fim, a Seção 6 é detalhada as conclusões deste artigo.

\section{Currículo de Referência}

Muitos cursos de Computação surgiram no Brasil nos anos 80 devido à crescente demanda de profissionais da área [Jonathan 2016]. Neste contexto, Currículos de Referência foram estruturados para garantir que egressos desses cursos atendessem a certas competências esperadas para o perfil de um profissional da área. Nos cursos de Computação, tais competências são definidas por associações de computação, tais como a SBC, a ACM (Association for Computing Machinery) e a IEEE Computer Society. A SBC, com base no currículo internacional proposto pela ACM e IEEE para as áreas de Computação, definiu a base curricular de Computação no Brasil em 1991 [Jonathan 2016].

Atualmente, o CR da SBC [SBC 2017] é resultado de debates em diversos de seus eventos, sendo o Workshop sobre Educação em Computação (WEI) o principal deles. Em 2016, foram homologados novos CRs para cada um dos cursos de computação: Ciência da Computação, Engenharia de Computação, Engenharia de Software, Licenciatura em Computação e Sistemas de Informação. O trabalho foi conduzido pelos membros da Comissão de Educação de forma a atender as Diretrizes Curriculares Nacionais (DCNs) e seguindo um modelo baseado em competências o qual foi inspirado na Taxonomia de Bloom Revisada [Ferraz et al. 2010]. Segundo o CR da SBC [SBC 2017], conhecimento e habilidades esperadas pelos alunos são definidas como competências.

Com relação à estrutura, o CR atual da SBC é composto por eixos de formação que abrangem o perfil esperado do egresso de cada curso. Cada um dos eixos corresponde a uma macro competência que se relaciona a grupos de competências derivadas (competências e habilidades oriundas das Diretrizes Curriculares Nacionais [MEC 2012]). 
Este trabalho se concentra no CR de Ciência da Computação, que possui os eixos a seguir:

Eixo 1: Resolução de Problemas. Corresponde à competência do egresso de resolver problemas, os quais devem possuir solução algorítmica.

Eixo 2: Desenvolvimento de Sistemas. Corresponde à competência sobre o desenvolvimento de sistemas de qualidade, seja do processo de criação ou do produto final.

Eixo 3: Desenvolvimento de Projetos. Representa a competência do egresso de desenvolver quaisquer tipos de projetos em equipes multidisciplinares.

Eixo 4: Implantação de Sistemas. Representa a competência de implantação de sistemas computacionais de forma a garantir a consistência e considerando o seu planejamento, execução e capacitação das pessoas envolvidas;

Eixo 5: Gestão de Infraestrutura. Corresponde ao gerenciamento de toda a infraestrutura computacional, incluindo o planejamento, implantação e manutenção do projeto;

Eixo 6: Aprendizado Contínuo e Autônomo. Consiste na capacidade do egresso de aprender por conta própria acerca de métodos, instrumentos, tecnologias de infraestrutura e domínios de aplicação da computação;

Eixo 7: Ciência, Tecnologia e Inovação. Representa a capacidade do egresso acerca do desenvolvimento de estudos avançados, sendo estes de classe científica e tecnológica ou para aplicação de soluções computacionais inovadoras.

O Apêndice A lista todos os 77 conteúdos do CR da SBC para a Ciência da Computação. Tais conteúdos aparecem de forma distribuída e não exclusiva entre os eixos e em suas competências derivadas. O Apêndice B, por sua vez, lista os conteúdos contidos em cada um dos eixos do CR. Ambos os apêndices destacam, em negrito, os conteúdos que apareceram nas edições do POSCOMP.

\section{POSCOMP}

O Exame Nacional para Ingresso na Pós-Graduação em Computação é organizado pela Sociedade Brasileira de Computação e tem como objetivo específico avaliar os conhecimentos em Computação dos candidatos a Programas de Pós-Graduação em Computação oferecidos no Brasil. O exame começou a ser aplicado no Brasil em 2002 [SBC 2018].

Boa parte dos programas de Pós-Graduação em Computação do Brasil utiliza o resultado do POSCOMP como um dos critérios de classificação no processo seletivo e para concessão de bolsas de estudo. Isso permite ao candidato a possibilidade de participar de processos seletivos em diversos programas de Pós-Graduação no país, sem a necessidade de se deslocar até as respectivas universidades para participar da seleção.

O POSCOMP é realizado por meio de uma prova teórico-objetiva, composta por 70 questões de múltipla escolha, subdividida em três áreas do conhecimento: 20 questões para área de Matemática; 30 para a área de Fundamentos da Computação; e 20 questões para a área de Tecnologia da Computação. Conforme [SBC 2016], os conteúdos abordados pelo POSCOMP baseiam-se no CR da SBC para a Ciência da Computação.

\section{Metodologia de Análise}

A análise realizada compara os conteúdos abordados nas sete últimas edições do POSCOMP (de 2014 a 2019) com a mais recente versão do currículo de referência da SBC [SBC 2017]. Dessa forma, pretende-se identificar o grau de cobertura da prova em relação 
ao temas que devem ser abordados em um curso de Ciência da Computação. Para avaliar a cobertura de conteúdos, os exames do POSCOMP foram analisados considerando como cada questão se relaciona aos conteúdos e aos eixos de formação do CR.

Para a análise orientada por conteúdos, realizou-se o cruzamento entre os conteúdos das questões dos exames e todos os conteúdos do CR. Com isso, é possível observar o grau de cobertura da prova em um nível menos abstrato e ainda identificar os conteúdos considerados os mais importantes pelos organizadores da prova. Durante a análise das questões em relação aos conteúdos, a multidisciplinaridade não foi considerada, ou seja, uma questão pode estar relacionada a apenas um conteúdo.

Já na análise orientada por eixos, realizou-se o cruzamento dos eixos de formação com os conteúdos das questões por edição do POSCOMP. Diferentemente da análise anterior, a granularidade das informações é mais grossa, por considerar um maior nível de abstração. Tal cruzamento visa avaliar o grau de incidência dos conteúdos das provas nos diferentes eixos dos cursos de Computação, isto é, como o POSCOMP tem coberto os eixos do CR. A partir disso, foi possível identificar a incidência de cada um dos eixos e destacar o de maior incidência, isto é, o eixo que o POSCOMP mais tem explorado.

Por fim, foi verificada e discutida a incidência de cada eixo e conteúdo no exame do POSCOMP, a qual definiu-se como a frequência de questões por eixo/conteúdo em cada uma das provas. Além disso, a análise permite identificar quais conteúdos do CR não foram cobertos pela prova até então e, portanto, apresentaram incidência 0 .

\section{Resultados}

Nesta seção, são apresentados os resultados das análises de correspondência entre as questões das edições do POSCOMP e os conteúdos do CR. Nos gráficos, foram considerados apenas os conteúdos que obtiveram incidência maior que 0 em alguma edição do exame. Primeiramente, são apresentados os resultados referentes aos graus de incidência de cada um dos eixos de formação do CR nas questões das provas analisadas. Em seguida, são apresentados os resultados da ocorrência de cada conteúdo do CR nas provas.

\subsection{Incidência de Eixos de Formação por Edição}

A Figura 1 mostra a incidência de eixos de formação do CR por edição do POSCOMP, exibindo a relevância de cada eixo em termos de número de questões.

Ao longo do período observado, o eixo que possui o maior número de questões abordando seus conteúdos é o Eixo 1 (Resolução de Problemas). Acredita-se que a importância deste eixo se deve ao fato de que a identificação de problemas e propostas de solução através de meios algorítmicos está presente durante toda careira do profissional de Computação. Isso pode ser refletido também pelo fato de tal eixo ser o segundo que mais contempla conhecimentos no CR. Considerando o mesmo princípio, encontram-se, por ordem decrescente de influência, o Eixo 2 (Desenvolvimento de Sistemas), Eixo 7 (Ciência, Tecnologia e Inovação), Eixo 5 (Gestão de Infraestrutura), Eixo 4 (Implantação de Sistemas), Eixo 3 (Desenvolvimento de Projetos) e por último o Eixo 6 (Aprendizado Contínuo e Autônomo). Este último eixo apresenta pouca influência em todas as edições analisadas. Os Eixos 1 e 2 apresentam comportamentos semelhantes no decorrer dos anos. O mesmo pode ser identificado para os pares de Eixos 4-5 e 3-6. Estes comportamentos análogos ocorrem devido aos conteúdos compartilhados pelos eixos comparados. 


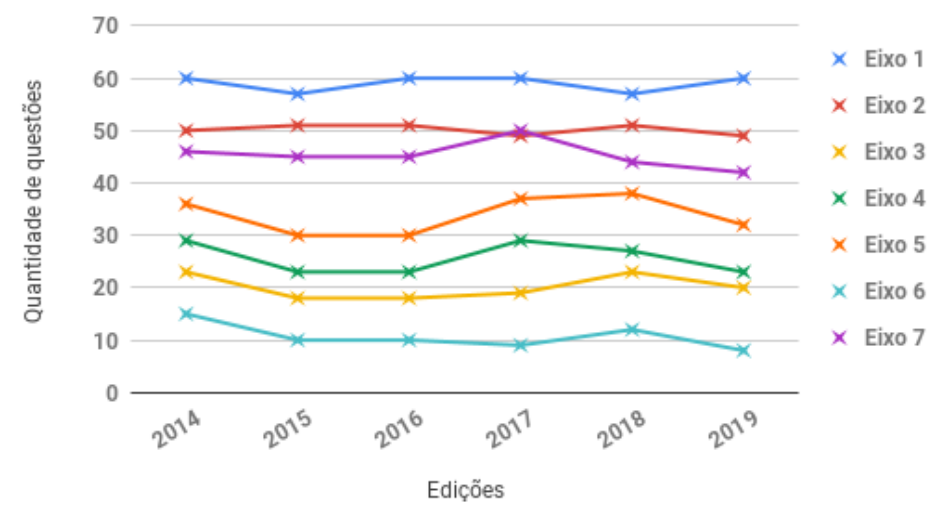

Figura 1. Gráfico da incidência de Eixos de Formação por edição

A Figura 2 apresenta os gráficos de incidência de conteúdos por ano, considerando cada um dos eixos de formação do CR. A partir destes gráficos, é possível fazer uma análise mais fina acerca dos conteúdos abordados em cada um dos exames do POSCOMP. O Eixo 1, que é composto por 42 conteúdos, teve 23 destes contemplados no período, aproximadamente 54,8\%, enquanto que o Eixo 3 apresentou apenas 8 conteúdos dos seus 20 totais, isto é, $40 \%$. Além disso, alguns eixos foram pouco contemplados, como o Eixo 6 que contabilizou apenas 3 conteúdos de um total de 13, ou seja, apenas $23,1 \%$.

Observando as Figuras 1 e 2, é possível inferir que, em geral, o grau de influência dos eixos é relacionado diretamente à quantidade de conteúdos que eles contemplam. As exceções a este comportamento ocorrem nos pares de Eixos 1 e 2, e 3 e 4: embora o Eixo 2 seja o que contemple mais conhecimentos, o Eixo 1 ainda apresenta uma cobertura maior de questões devido ao fato de que muitos conhecimentos do Eixo 2 têm incidência igual a 0 ; o Eixo 3 possui 8 conteúdos contemplados em todo o período, embora tenha menor influência do que o Eixo 4, que possui 7. Especificamente, este fato decorre do grau de incidência dos conteúdos, ou seja, os conteúdos do Eixo 4 aparecem em maior quantidade do que os do Eixo 3. Note que o Eixo 6 não contém conteúdos exclusivos (não cobertos por outros eixos). Desta forma, o Eixo 6 não apresenta influência exclusiva.

\subsection{Incidência de Conteúdo por Questões}

A Figura 3 apresenta a incidência de conteúdos do CR contemplados por edição do POSCOMP sem distinguir eixos. Nesta figura, quanto maior o círculo, maior a incidência de questões de um conteúdo. Apenas 30 dos 77 conteúdos do CR são exibidos $(38,9 \%)$, correspondendo àqueles com ao menos 1 questão em alguma edição do exame no período de interesse. Identificou-se, ainda, que os conteúdos C03 (Arquitetura e Organização de Computadores) e C73 (Sistemas Operacionais) apresentaram os maiores números de questões. Por outro lado, os conteúdos C28 (Gerenciamento de Projetos), C45 (Padrões de Projeto) e C60 (Projeto de algoritmos) apresentam apenas uma questão em todo o período avaliado.

Com o objetivo de avaliar a incidência dos conteúdos, estes foram ordenados de acordo com o somatório de questões contabilizadas nas edições 2014-2019. Os conteúdos com um somatório de questões acima da mediana (18,5) foram: C03, C73, C48, C02, C77, C09, C38, C65, C39, C76, C27, C58, C01, C21, C08 e C12. 

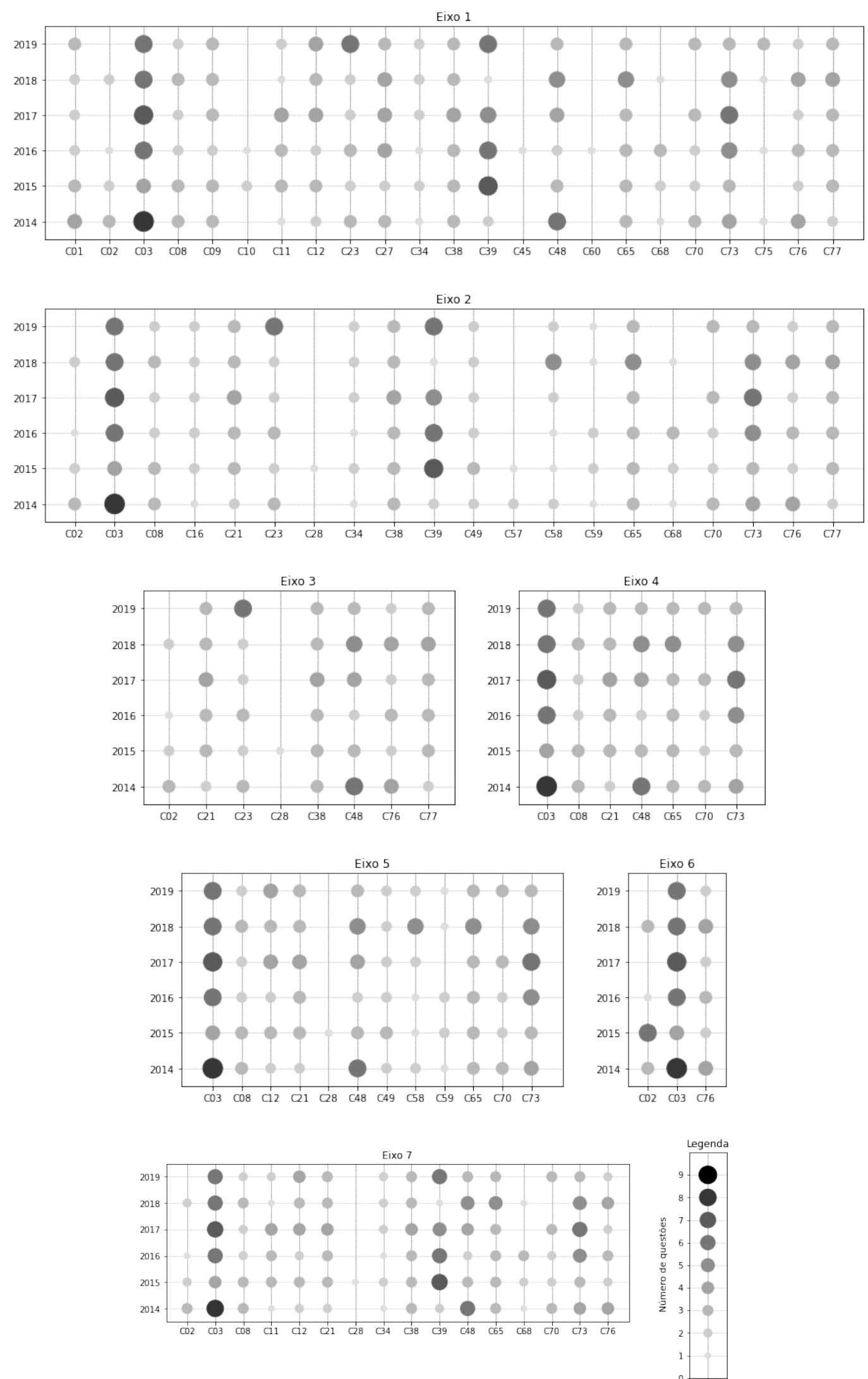

Figura 2. Gráficos de incidência de conteúdos por eixo do CR 

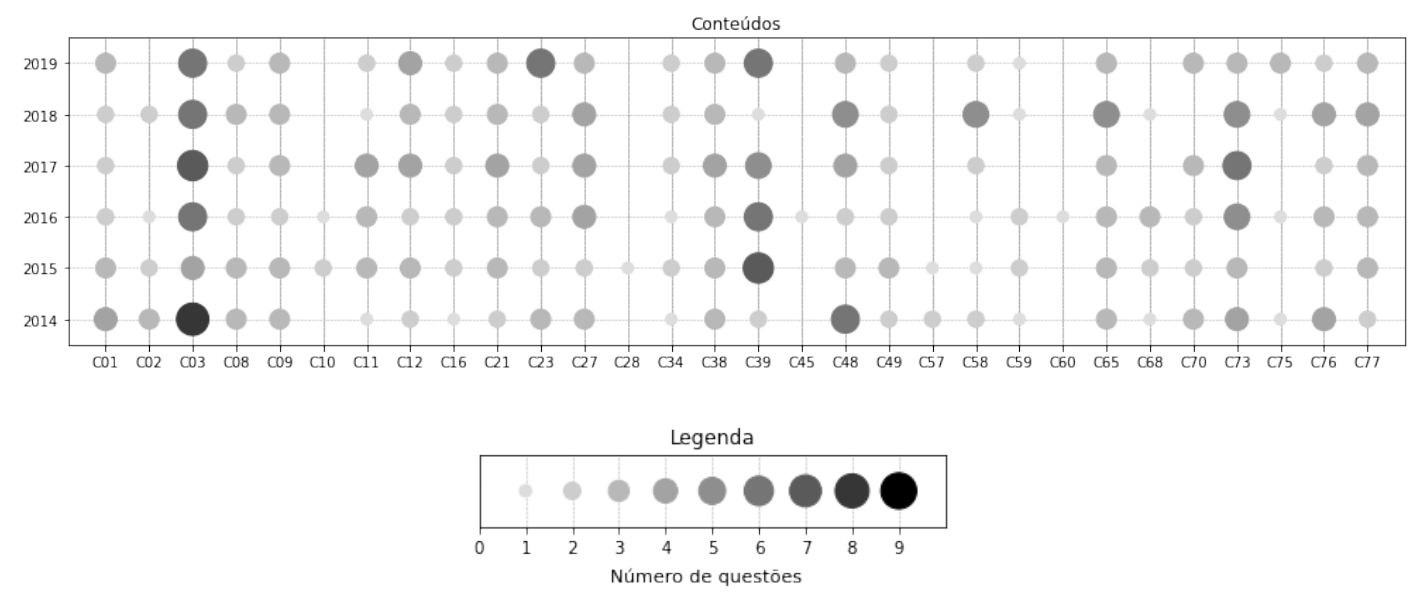

Figura 3. Gráfico de incidência de questões por conteúdo do CR

Em relação à consistência, observou-se que 22 conteúdos apresentaram desvio padrão inferior à 1 , indicando uma baixa variação de cobertura ao longo das edições. Esses foram: C01, C08, C09, C10, C12, C16, C21, C27, C28, C34, C38, C45, C49, C50, C56, C57, C59, C60, C65, C70, C76 e C77. Já os que obtiveram um desvio padrão inferior à mediana 0,74 foram, em ordem crescente de desvio: C50, C56, C09, C16, C28, C38, C45, C49, C08, C34, C60, C27, C70, C59, e C77. Por outro lado, os conteúdos C02 (Algoritmos) e C39 (Matemática Discreta) apresentaram um comportamento oscilante bastante significativo, com desvio padrão superior a 2 (respectivamente, 2,11 e 2,16). Tal comportamento pode demonstrar a intenção dos elaboradores em equilibrar a exploração dos conteúdos ao longo das edições, balanceando-os.

Combinando as informações de incidência e consistência, é possível identificar os conteúdos mais influentes, ou seja, que possuem tanto um alto índice de incidência quanto uma frequência regular ao longo dos anos. Os mais influentes são: C03 (Arquitetura e Organização de Computadores) e C73 (Sistemas Operacionais). Em contrapartida, foram contabilizados 47 que não possuem influência alguma por não se apresentarem em nenhuma das 6 edições do POSCOMP. São eles: C04, C05, C06, C07, C13, C14, C15, C17, C18, C19, C20, C22, C24, C25, C26, C29, C30, C31, C32, C33, C35, C36, C37, C40, C41, C42, C43, C44, C46, C47, C50, C51, C52, C53, C54, C55, C56, C61, C62, C63, C64, C66, C67, C69, C71, C72 e C74.

Como a versão do CR utilizada nesta análise foi homologada no final de 2016, sua vigência contempla apenas as edições de 2017 e 2019 do POSCOMP. Diante desse fato, realizou-se uma análise considerando apenas estes três anos. Tal análise mostrou que 26 conteúdos foram abordados. Não obstante, todos eles foram explorados entre 2014 e 2016. Além disso, os 6 conteúdos que não foram contemplados em 2017 e 2019, já apresentavam baixa incidência nas edições anteriores: C10, C28, C45, C50, C56 e C57.

\section{Conclusão}

Este trabalho apresenta uma análise comparativa entre o Currículo de Referência da SBC e as questões do exame nacional, POSCOMP. Foram identificados os conteúdos do CR que aparecem com maior frequência e quais são seus comportamentos. Ao longo do tempo, foi identificado também que mais de $60 \%$ dos conteúdos do CR não são contemplados e 
como cada eixo de formação é explorado na construção do exame. Além disso, a análise conduzida indica que o POSCOMP prioriza alguns conteúdos do $\mathrm{CR}$ em detrimento de outros. Com isso, é possível concluir que a SBC considera que tais conteúdos do CR são os mais relevantes aos egressos.

Os interessados em realizar o POSCOMP e ingressar em cursos de pós-graduação podem se beneficiar dos resultados produzidos nesta análise. Há também contribuição aos elaboradores da prova, oferecendo identificação da cobertura dos conteúdos do CR no exame e destacando-os em cada um dos eixos de formação, bem como aos professores, que podem utilizar os resultados do estudo para dar enfoque aos assuntos dos conteúdos do CR com maior incidência. Por fim, os coordenadores de cursos de graduação podem adequar, caso julguem necessário, o currículo dos respectivos cursos de forma a adaptá-lo ao propósito que a SBC tem no planejamento do CR e na aplicação do POSCOMP.

Este trabalho pode ser estendido com a classificação das questões do exame nos seis níveis do processo cognitivo da Taxonomia de Bloom Revisada [Ferraz et al. 2010]. Outras extensões podem incluir uma análise considerando a diferenciação de conteúdos por ênfases da Computação (Teoria da Computação, Inteligência Artificial, Sistemas Embarcados, Engenharia de Software, Métodos Formais, entre outras) ou a verificação de como os desempenhos do Exame Nacional de Desempenho dos Estudantes (ENADE) se relacionam aos do POSCOMP.

\section{Referências}

Ferraz, A., Belhot, R. V., et al. (2010). Taxonomia de bloom: revisão teórica e apresentação das adequações do instrumento para definição de objetivos instrucionais. Gest. Prod., São Carlos, 17(2):421-431.

Jonathan, M. (2016). Currículos de computação: porque permanecem assim. In WEI-24 ${ }^{o}$ Workshop sobre Educação em Computação-XXXVI Congresso da Sociedade Brasileira de Computação, Março.

Junior, F. D. S. and Brancher, J. (2014). Uma pesquisa de opinião sobre a relevância dos conteúdos abrangidos pelo poscomp. In Anais do XXII Workshop sobre Educação em Computação, pages 90-99, Porto Alegre, RS, Brasil. SBC.

MEC (2012). MEC/CNE/CES Diretrizes Curriculares Nacionais para os cursos de graduação em Computação, Parecer CNE/CES no. 136/2012. Ministério da Educação. Disponível em: http://portal.mec.gov.br, acesso em: 05/11/2021.

SBC (2016). Aumentam em 20\% as inscrições para o POSCOMP 2016 promovido pela SBC. Disponível em: http://www.sbc.org.br, acesso em: 05/11/2021.

SBC (2017). Referênciais de Formação para Cursos de Graduação em Computação, Proposta versão 2017. Diretoria de Educação, Sociedade Brasileira de Computação. Disponível em: http://www.sbc.org.br, acesso em: 05/11/2021.

SBC (2018). Poscomp. Disponível em: http://www.sbc.org.br, acesso em: 05/11/2021. 


\section{Apêndice A}

* Somente os conteúdos destacados (em negrito) apareceram no exame.

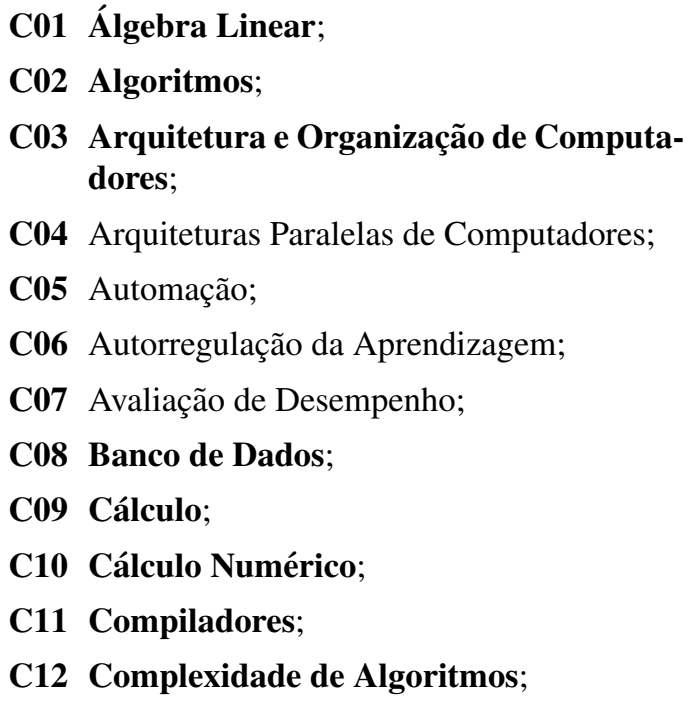

C03 Arquitetura e Organização de Computadores;

C04 Arquiteturas Paralelas de Computadores;

C05 Automação;

C06 Autorregulação da Aprendizagem;

C07 Avaliação de Desempenho;

C08 Banco de Dados;

C09 Cálculo;

C10 Cálculo Numérico;

C11 Compiladores;

C12 Complexidade de Algoritmos;

C13 Comportamento Humano nas Organizações;

C14 Computação e Sociedade;

C15 Computação em Nuvem;

C16 Computação Gráfica;

C17 Comunicação Profissional;

C18 Criptografia;

C19 Dependabilidade;

C20 Empreendedorismo;

C21 Engenharia de Software;

C22 Ergonomia;

C23 Estruturas de Dados;

C24 Ética em Computação;

C25 Fundamentos de Administração;

C26 Fundamentos de Economia;

C27 Geometria Analítica;

C28 Gerenciamento de Projetos;

C29 Gestão de Carreira Profissional;

C30 Gestão de Tempo;

C31 História da Computação;

C32 Inglês Instrumental;

C33 Inglês Técnico;

C34 Inteligência Artificial e Computacional;

C35 Interação Humano-Computador;

C36 Internet das Coisas (IoT);

C37 Legislação de Informática;

C38 Lógica Matemática;

C39 Matemática Discreta;
C40 Meio Ambiente;

C41 Metodologia Científica;

C42 Métodos Formais;

C43 Métodos Quantitativos em Computação;

C44 Modelagem de Sistemas;

C45 Padrões de Projetos;

C46 Pesquisa Operacional e Otimização;

C47 Privacidade e Direitos Civis;

C48 Probabilidade e Estatística;

C49 Processamento de Imagens;

C50 Processamento Distribuído;

C51 Processamento Paralelo;

C52 Programação de Aplicações Web;

C53 Programação de Aplicativos para Dispositivos Móveis;

C54 Programação em Linguagem de Montagem;

C55 Programação em Linguagem Script;

C56 Programação em Lógica;

C57 Programação Funcional;

C58 Programação Imperativa;

C59 Programação Orientada a Objetos;

C60 Projeto de Algoritmos;

C61 Projeto de Sistemas Computacionais;

C62 Propriedade Intelectual;

C63 Realidade Virtual e Aumentada;

C64 Recuperação da Informação;

C65 Redes de Computadores;

C66 Segurança de Sistemas Computacionais;

C67 Simulação de Sistemas;

C68 Sistemas Concorrentes;

C69 Sistemas de Tempo Real;

C70 Sistemas Distribuídos;

C71 Sistemas Embarcados;

C72 Sistemas Multimídia;

C73 Sistemas Operacionais;

C74 Sustentabilidade;

C75 Técnicas de Programação;

C76 Teoria da Computação;

C77 Teoria dos Grafos. 


\section{Apêndice B}

* Somente os conteúdos destacados (em negrito) apareceram no exame.

Tabela 1. Eixos de formação e seus conteúdos associados

\begin{tabular}{|c|c|}
\hline & Conteúdos \\
\hline $\begin{array}{c}\text { Eixo } 1 \\
\text { (Resolução de Problemas) }\end{array}$ & 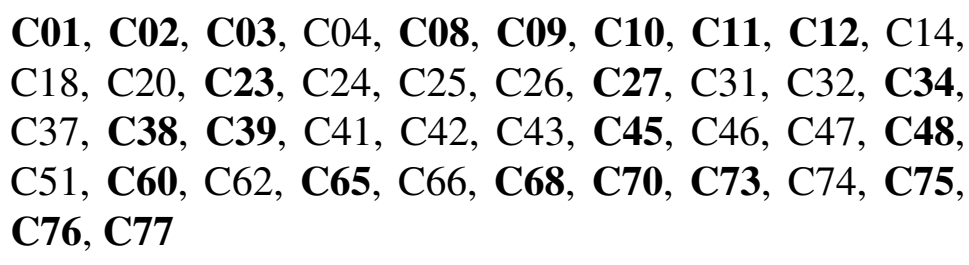 \\
\hline $\begin{array}{l}\text { Eixo } 2 \\
\text { (Desenvolvimento de } \\
\text { Sistemas) }\end{array}$ & $\begin{array}{l}\text { C02, C03, C05, C07, C08, C13, C14, C15, C16, C17, } \\
\text { C18, C19, C21, C23, C24, C28, C32, C34, C } 35, \text { C } 36, \\
\text { C } 37, \mathbf{C 3 8 , ~ C 3 9 , ~ C 4 0 , ~ C 4 2 , ~ C 4 3 , ~ C 4 9 , ~ C 5 0 , ~ C 5 1 , ~ C 5 2 , ~} \\
\text { C53, C56, C57, C58, C59, C63, C64, C65, C66, C68, } \\
\text { C69, C70, C71, C72, C73, C74, C76, C77 }\end{array}$ \\
\hline $\begin{array}{l}\text { Eixo } 3 \\
\text { (Desenvolvimento de } \\
\text { Projetos) }\end{array}$ & $\begin{array}{l}\text { C02, C07, C13, C14, C17, C20, C21, C23, C24, C28, } \\
\text { C32, C33, C37, C38, C40, C42, C43, C48, C76, C77 }\end{array}$ \\
\hline $\begin{array}{c}\text { Eixo } 4 \\
\text { (Implantação de Sistemas) }\end{array}$ & $\begin{array}{l}\text { C03, C07, C08, C13, C14, C17, C21, C24, C25, C26, } \\
\text { C31, C32, C37, C40, C43, C44, C47, C48, C62, C65, } \\
\text { C66, C67, C70, C73, C74 }\end{array}$ \\
\hline $\begin{array}{c}\text { Eixo } 5 \\
\text { (Gestão de Infraestrutura) }\end{array}$ & $\begin{array}{l}\text { C03, C07, C08, C12, C13, C14, C15, C17, C18, C19, } \\
\text { C21, C22, C24, C25, C28, C } 31, \mathrm{C} 32, \mathrm{C} 35, \mathrm{C} 36, \mathrm{C} 37, \\
\mathrm{C} 40, \mathrm{C} 43, \mathrm{C} 44, \mathrm{C} 47, \mathbf{C} 48, \mathrm{C} 49, \mathrm{C} 51, \mathrm{C} 54, \mathrm{C} 55, \mathrm{C} 58, \\
\text { C59, C61, C62, C64, C65, C66, C67, C69, C70, C } 71, \\
\text { C72, C73, C74 }\end{array}$ \\
\hline $\begin{array}{c}\text { Eixo } 6 \\
\text { (Aprendizado Contínuo e } \\
\text { Autônomo) }\end{array}$ & $\begin{array}{l}\text { C02, C03, C06, C17, C29, C30, C32, C } 33, \mathrm{C} 41, \mathrm{C} 43, \\
\mathrm{C} 44, \mathrm{C} 67, \mathbf{C} 76\end{array}$ \\
\hline $\begin{array}{l}\text { Eixo } 7 \\
\text { (Ciência, Tecnologia e } \\
\text { Inovação) }\end{array}$ & $\begin{array}{l}\text { C02, C03, C04, C07, C08, C11, C12, C14, C21, C24, } \\
\text { C28, C34, C } 35, \mathrm{C} 37, \mathbf{C 3 8}, \mathbf{C 3 9}, \mathrm{C} 40, \mathrm{C} 41, \mathrm{C} 42, \mathrm{C} 43, \\
\text { C47, C48, C51, C61, C62, C65, C66, C68, C70, C73, } \\
\text { C76 }\end{array}$ \\
\hline
\end{tabular}

